\title{
TLC-Densitometric Determination of Sulfasalazine and Its Possible Impurities in Pharmaceutical Preparations
}

\author{
A. KWIECIEN' ${ }^{1}{ }^{*},{ }^{\prime}$ K. PIĄTEK ${ }^{1}$, P. ŻMUDZKI ${ }^{2}$, AND J. KRZEK ${ }^{1}$ \\ ${ }^{1}$ Department of Inorganic and Analytical Chemistry, Jagiellonian University, Medical College, \\ Faculty of Pharmacy, Medyczna 9, 30-688 Kraków, Poland \\ 2Department of Medicinal Chemistry, Jagiellonian University, Medical College, \\ Faculty of Pharmacy, Medyczna 9, 30-688 Kraków, Poland \\ *E-mail: annakwiecien@op.pl
}

Summary. A simple, selective, and sensitive thin-layer chromatographic-densitometric method has been developed for the determination of sulfasalazine besides its possible impurities in pharmaceutical preparations. The mobile phase was composed of ethyl acetate-methanol-ammonia $25 \% 10: 7: 3(v / v / v)$, and the stationary phase was aluminum plates precoated with silica gel $60 \mathrm{~F}_{254}$ that enabled to obtain well resolved peaks of sulfasalazine and its impurities. The developed chromatograms were analyzed densitometrically at $\lambda=360 \mathrm{~nm}$. $R_{\mathrm{F}}$ values and ultraviolet (UV) spectra were used to identify the compounds. The developed method is highly sensitive (limit of detection [LOD] $=17.11$ ng spot ${ }^{-1}$, limit of quantitation [LOQ] $=51.84 \mathrm{ng} \mathrm{spot}^{-1}$ ), precise (relative standard deviation $[\mathrm{RSD}]=1.43 \%-4.28 \%)$, and accurate $(\mathrm{RSD}=1.64 \%-4.27 \%)$. The linearity of the method was checked within the range $20-120 \mathrm{ng} \mathrm{spot}^{-1}$. The method was successfully applied for the determination of sulfasalazine in pharmaceutical preparations besides its impurities. The structures of impurities present in the standard substance and in pharmaceutical preparations were established by ultra-performance liquid chromatographytandem mass spectrometry (UPLC-MS/MS) technique.

Key Words: thin-layer chromatography, drug analysis, sulfasalazine, impurities

\section{Introduction}

Sulfasalazine 5-[4-(2-pyridylsulfamoyl)phenylazo]salicylic acid was designed by Svartz in 1942 by combining an antibiotic sulfapyridine with an anti-inflammatory agent 5 -aminosalicylic acid through an azo bond [1]. Sulfasalazine is a bright-yellow or brownish-yellow fine powder, practically insoluble in water, very slightly soluble in alcohol, practically insoluble in methylene chloride, but well soluble in dilute solutions of alkali hydroxides [2]. Sulfasalazine is commonly used in the treatment of inflammatory bowel diseases such as rheumatoid arthritis, Crohn's disease and ulcerative colitis [3]. Sulfasalazine can reverse the scarring associated with cirrhosis of the liver. Myofibroblasts cells contribute to scar tissue in a diseased liver; these cells also secrete proteins that prevent the breakdown of the scar tissue. Sulfasalazine retards this secretion. It also helps in healing cirrhosis of the liver 
[4]. However, it can cause adverse effects which may lead to disorder of the hemopoietic system and potential carcinogenic effects. Different analytical methods were applied for the determination of sulfasalazine in pharmaceutical preparations and biological fluids. For the assessment of sulfasalazine in several biological matrices, an enzyme-linked immunosorbent assay method based on polyclonal antibodies was developed and characterized [1]. Ultraviolet (UV) spectrophotometric method was applied for the quantitative determination of sulfasalazine in tablet dosage form [5]. Two kinetic spectrophotometric methods were developed for the determination of sulfasalazine in pure form and in pharmaceutical formulations. The first method was based upon the kinetic investigation of the oxidation reaction of sulfasalazine with alkaline potassium permanganate at room temperature for fixed time yielding a green color of manganate ion. The second method was based upon the oxidation reaction of sulfasalazine with a mixture of iodine and potassium iodide at room temperature [6]. Sulfasalazine and its main metabolites sulphapyridine and 5-aminosalicylic acid were determined in human plasma by liquid chromatography-mass spectrometry (LC-MS) method using XBP Phenyl column [7]. Sulfasalazine was also determined in pharmaceutical and biological samples using molecularly imprinted polymer modified carbon paste electrode. Trace amount of sulfasalazine was determined by using differential pulse voltammetry method based on electrochemical reduction of sulfasalazine at the modified electrodes [8]. An analytical method based on capillary electrophoresis and tandem mass spectrometry was proposed and validated for the identification and simultaneous quantification of 12 sulfonamides including sulfasalazine in pork meat [9].

The aim of this study was to develop a chromatographic-densitometric method for the determination of sulfasalazine besides its possible impurities in pharmaceutical preparations. It has been shown that this technique has many advantages, such as providing an accurate, quick, and simple determination and can be an alternative to the commonly used LC methods. The determined impurities were also subjected to ultra-performance liquid chromatography-tandem mass spectrometry (UPLC-MS/MS) analysis to establish their chemical structures.

\section{Experimental}

\section{Chemicals and Preparations}

- Standard substance: sulfasalazine - 5-[4-(2-pyridylsulfamoyl)phenylazo]salicylic acid (Sigma-Aldrich $\mathrm{GmbH}$, St. Louis, MO, USA) 
- Chemical reagents: ethyl acetate (POCh), methanol (Merck), ammonia 25\% (POCh), water (Merck), acetonitrile (Merck), formic acid (Merck), and sodium hydroxide (POCh). All reagents were of analytical grade

- Pharmaceutical preparations: Sulfasalazin EN 500 mg (Krka, Novo mesto, Slovenia) and Salazopyrin EN $500 \mathrm{mg}$ (Pfizer Health AB, Stockholm, Sweden)

\section{Solutions for Analysis}

Standard solutions were prepared by dissolving an appropriate amount of standard substance of sulfasalazine in $0.1 \mathrm{~mol} \mathrm{~L}^{-1} \mathrm{NaOH}$ to give a concentration of $0.1 \%(w / v)$. Then, the solution was diluted with the same solvent to obtain a concentration of $0.001 \%(w / v)$ for linearity and precision studies. Standard solution of sulfasalazine at a concentration of $0.1 \%(w / v)$ was used for the purity studies.

Preparation solutions were prepared by grinding ten tablets of a pharmaceutical preparation in a mortar, weighing with a precision of $0.1 \mathrm{mg}$ the amount of tablet powder corresponding to $25 \mathrm{mg}$ of sulfasalazine and shaking for 30 min with $15 \mathrm{~mL}$ of $0.1 \mathrm{~mol} \mathrm{~L}^{-1} \mathrm{NaOH}$ in a $25-\mathrm{mL}$ volumetric flask. Then the flask was filled with $0.1 \mathrm{~mol} \mathrm{~L}^{-1} \mathrm{NaOH}$ to the specified volume and placed in an ultrasonic bath for $30 \mathrm{~min}$. After that, the solution was filtered and diluted with $0.1 \mathrm{~mol} \mathrm{~L}^{-1} \mathrm{NaOH}$ to obtain a concentration of $0.001 \%(w / v)$.

\section{Chromatography}

Standard solution of sulfasalazine was applied in $8 \mathrm{~mm}$ bands, $10 \mathrm{~mm}$ apart, and $10 \mathrm{~mm}$ from the lower edge to thin-layer chromatography (TLC) silica gel $60 \mathrm{~F}_{254}$ plates $(65 \times 100 \mathrm{~mm})$ using CAMAG sample applicator Linomat 4 equipped with a $100-\mu \mathrm{L}$ syringe at a constant application rate of $200 \mathrm{~nL} \mathrm{~s}^{-1}$. Chromatograms were developed over a distance of $95 \mathrm{~mm}$ using ethyl acetate-methanol-ammonia $25 \%$ 10:7:3 $(v / v / v)$ in a chromatographic chamber $18 \times 9 \times 18 \mathrm{~cm}$ in size (Sigma-Aldrich, USA) saturated for $10 \mathrm{~min}$ with the mobile phase vapour at room temperature. The mobile phase was chosen experimentally after checking 20 different mobile phases. After development, the TLC plates were dried at room temperature and scanned at $360 \mathrm{~nm}$, which was chosen on the basis of an absorption spectrum recorded directly from the chromatogram. The analytical wavelength was the same for sulfasalazine and its impurities. Densitometric scanning was carried out using a TLC Scanner 3 (CAMAG, Switzerland) controlled by win CATS 1.3.4 software resident in the system in the linear absorbance-reflectance 
mode. The slit dimensions were $8 \times 0.6 \mathrm{~mm}$, the scanning speed was 20 $\mathrm{mm} \mathrm{s}^{-1}$, and data resolution was $100 \mu \mathrm{m} \mathrm{step}^{-1}$. Retardation factors $\left(R_{\mathrm{F}}\right.$ values) were used to identify sulfasalazine and its possible impurities. Good separation and well-developed peaks were obtained in a short time (approximately $30 \mathrm{~min}$ ).

\section{Validation of the Method}

The optimized method was validated for the determination of sulfasalazine in the presence of its impurities according to the International Conference on Harmonization (ICH) guidelines [10, 11].

\section{Specificity}

The specificity of the method was ascertained by comparing chromatograms of sulfasalazine obtained for standard solution, preparation solution, and blank chromatogram. In the obtained chromatograms, the retardation factor $\left(R_{\mathrm{F}}\right)$ values of analyzed substances, resolution factor, and peak areas were taken into account. Resolution factor $\left(R_{\mathrm{s}}\right)$ was calculated using the formula:

$R_{\mathrm{s}}=2 \times$ (distance between the centers of two adjacent spots)/(sum of the two spots in the direction of development).

\section{Linearity}

To check linearity of the method, $2,4,6,8,10$, and $12 \mu \mathrm{L}$ of sulfasalazine standard solution at a concentration of $0.001 \% w / v$ were applied to the chromatographic plate $120 \times 100 \mathrm{~mm}$ in size. Linearity was determined based on the relationship between the peak areas and sulfasalazine mass $\left(\right.$ ng spot $\left.^{-1}\right)$. The regression plot, regression equation, coefficient of determination $r^{2}$, and the distribution of residuals by Shapiro-Wilk normality test were calculated by Statistica 10.0 and are indicative of linearity.

\section{Precision}

The intra-day and inter-day precisions of the method were derived from the degree of consistency of the recorded peak areas for sulfasalazine standard solution. Six determinations were made by applying $60 \mathrm{ng} \mathrm{spot}^{-1}$ at $100 \%$ level, $30 \mathrm{ng}$ spot $^{-1}$ at $50 \%$ level, and $90 \mathrm{ng} \mathrm{spot}^{-1}$ at $150 \%$ level to the plate 
followed by the analysis according to the method conditions. The inter-day precision was carried out after 1 week by a different analyst. The precision was estimated using peak areas and was evaluated as the standard deviation (SD) and relative standard deviation (RSD) values.

\section{LOD and LOQ}

To determine the limit of detection (LOD) and limit of quantitation (LOQ), standard error of the estimate, the slope of the linear calibration plot, and the following equations were used: $\mathrm{LOD}=3.3 \times S_{\mathrm{e}} / a$ and $\mathrm{LOQ}=10 \times S_{\mathrm{e}} / a$, where $S_{\mathrm{e}}=$ standard error of the estimate and $a=$ slope of a straight line.

\section{Accuracy}

The accuracy of the method was determined by spiking the standard solution of sulfasalazine into sample solution in the amounts of $80-120 \%$ of the level in the preparations under examination. The obtained results were compared with the expected results and the recovery of the added drug was expressed as a percentage.

\section{Robustness}

To examine robustness, the most significant chromatographic parameters were changed within the range of $1-5 \%$ compared to those of the optimal conditions, while keeping the other parameters unchanged. The following parameters were examined: proportions of methanol and ammonia in the mobile phase, the mobile phase volume, the developing distance, saturation time of the chromatographic chamber. The influence of the stationary phase was also checked by application to HPTLC plates instead of TLC plates.

As a result of the determinations carried out, analysis conditions, which were later used to determine the sulfasalazine content in pharmaceutical preparations and to check its purity, were established.

\section{Identification of Possible Impurities by UPLC-MS/MS}

The UPLC-MS/MS system consisted of a Waters ACQUITY ${ }^{\circledR}$ UPLC ${ }^{\circledR}$ (Waters Corporation, Milford, MA, USA) coupled to a Waters TQD mass spectrometer (electrospray ionization mode electrospray ionization (ESI)tandem quadrupole). Chromatographic separations were carried out using the Acquity UPLC BEH (bridged ethyl hybrid) C18 column: $2.1 \times 100 \mathrm{~mm}$ 
and $1.7 \mu \mathrm{m}$ particle size. The column was maintained at $40{ }^{\circ} \mathrm{C}$ and eluted under gradient conditions from $95 \%$ to $0 \%$ of eluent A over $10 \mathrm{~min}$, at a flow rate of $0.3 \mathrm{~mL} \mathrm{~min}^{-1}$ (eluent $\mathrm{A}$ : formic acid-water [0.1\%, $\left.v / v\right]$; eluent $\mathrm{B}$ : formic acid-acetonitrile $[0.1 \%, v / v])$. One microliter of each sample was injected.

Chromatograms were made using Waters e $\lambda$ PDA detector. Spectra were analyzed in 200-700 $\mathrm{nm}$ range with $1.2 \mathrm{~nm}$ resolution and sampling rate of 20 points/s.

MS detection settings of Waters TQD mass spectrometer were as follows: source temperature, $150{ }^{\circ} \mathrm{C}$; desolvation temperature, $350{ }^{\circ} \mathrm{C}$; desolvation gas flow rate, $600 \mathrm{~L} \mathrm{~h}^{-1}$; cone gas flow, $100 \mathrm{~L} \mathrm{~h}^{-1}$; capillary potential, $3.00 \mathrm{kV}$; and cone potential, $20 \mathrm{~V}$. Nitrogen was used as both nebulizing and drying gas. The data were obtained in a scan mode ranging from 50 to 1000 $\mathrm{m} / \mathrm{z}$ at $0.5 \mathrm{~s}$ time intervals.

\section{Determination of Sulfasalazine and Its Impurities in Pharmaceutical Preparations}

In order to determine the sulfasalazine content, bands of solutions $(6 \mu \mathrm{L}$ of standard solution and preparation solution) were applied to the $120 \times$ $100 \mathrm{~mm}$ chromatographic plates of silica gel $60 \mathrm{~F}_{254}$ (cut from $20 \times 20 \mathrm{~cm}$ before use). The purity of the drugs was determined by using $20 \mu \mathrm{L}$ of the preparation solutions at a concentration of $1 \mathrm{mg} \mathrm{mL}^{-1}$. Chromatograms were developed to a distance of $95 \mathrm{~mm}$ with the experimentally chosen mobile phase and then dried at room temperature. Densitometric scanning was performed at $360 \mathrm{~nm}$, and peak areas were recorded. The concentration of sulfasalazine in preparations under examination was computed by comparing the peak areas for the standard and sample solutions. For quantitative determination of sulfasalazine impurities, the scan areas of appropriate peaks were recorded and the relative percent concentrations computed by employing the internal normalization method, according to the formula: $i[\%]=\left(x_{i} / \Sigma x\right) 100$, where $i[\%]$ is constituent concentration, $x_{i}$ is peak area for the determined constituent, and $\Sigma x$ is sum of peak scan areas in the chromatogram.

\section{Results and Discussion}

The aim of this paper was to establish a simple and accurate method for quantitative determination of sulfasalazine and its possible impurities in pharmaceutical preparations by a TLC-densitometric method. 


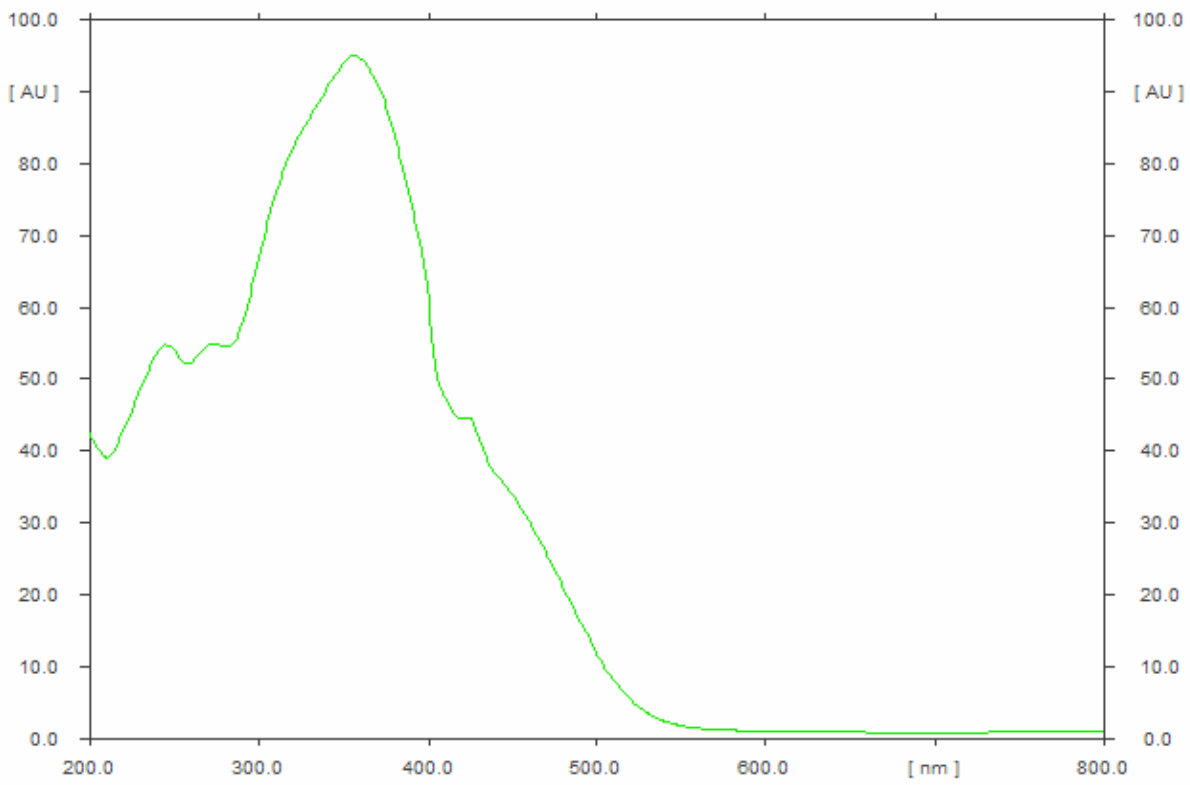

(A)

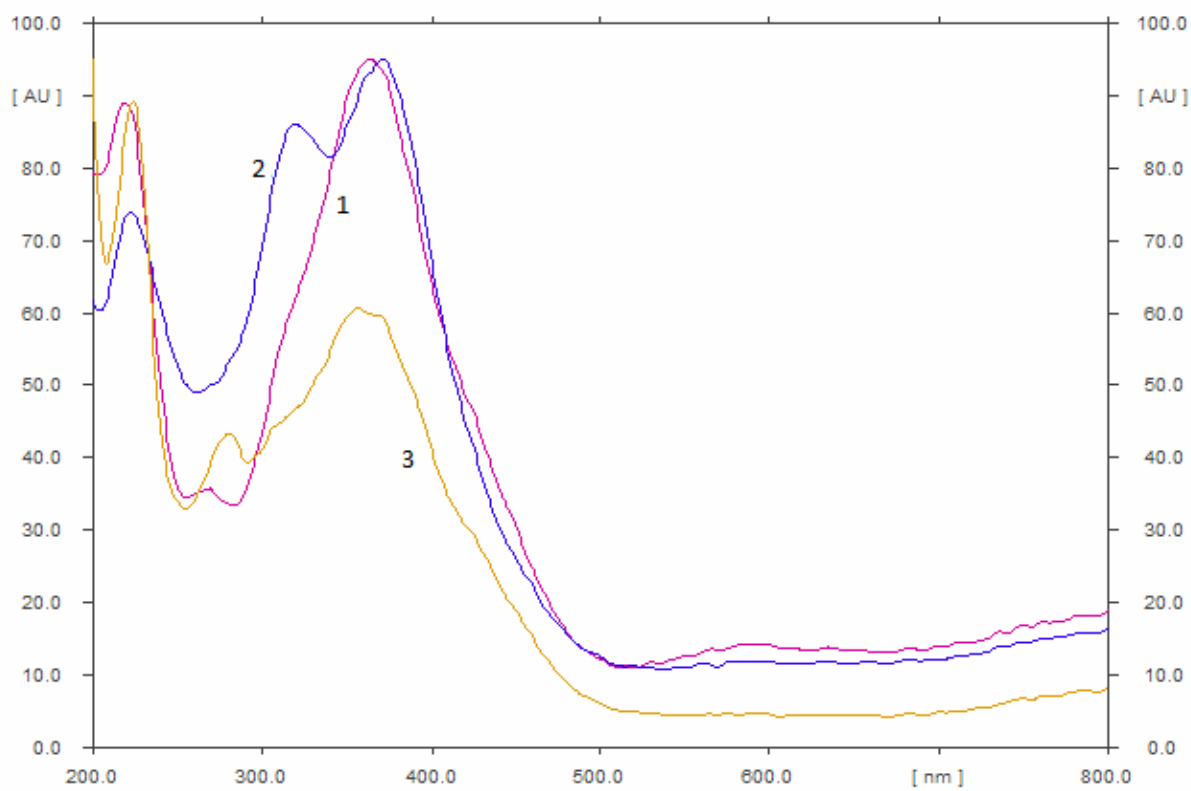

(B)

Fig. 1. Absorption spectra of sulfasalazine (A) and its impurities (B-1, 2, and 3) registered directly from the chromatogram 
By using TLC silica gel $60 \mathrm{~F}_{254}$ plates as a stationary phase and ethyl acetate-methanol-ammonia $25 \% 10: 7: 3(v / v / v)$ as the mobile phase, good separation of constituents under investigation was obtained. The eluent mixture was chosen experimentally after checking about 20 different mobile phases. Chromatograms were developed for $\sim 30 \mathrm{~min}$. The retardation factor for sulfasalazine was $R_{\mathrm{F}}=0.75$ and for impurities was as follows: 0.56 for impurity 1, 0.61 for impurity 2, and 0.88 for impurity 3 .

Registration of peak areas for sulfasalazine and its impurities was carried out in UV at $\lambda=360$ so that the simultaneous determination of sulfasalazine beside possible impurities was possible (Fig. 1).

Table I. Summary of the validation results for the determination of sulfasalazine

\begin{tabular}{|c|c|}
\hline Parameter & Result \\
\hline$R_{\mathrm{F}}$ & 0.75 \\
\hline Linearity & $\begin{array}{c}\text { Linearity range }\left(\text { ng spot }^{-1}\right): \\
20-120 \\
P=a c+b \\
a=35.218 ; b=391.08 \\
S_{a}=2.18 ; S_{b}=169.96 \\
r=0.9924 \\
\text { Shapiro-Wilk normality test: } \\
W=0.94204 ; p=0.67568\end{array}$ \\
\hline LOD (ng spot $\left.{ }^{-1}\right)$ & 17.11 \\
\hline LOQ (ng spot $\left.{ }^{-1}\right)$ & 51.84 \\
\hline $\begin{array}{c}\text { Accuracy }(\%) \\
n=5\end{array}$ & $\begin{array}{c}\text { Level } 80 \%: x_{\text {mean }}=98.31 \\
S=4.20 \\
\text { RSD }=4.27 \% \\
\text { Level } 100 \%: x_{\text {mean }}=101.43 \\
S=1.66 \\
\text { RSD }=1.64 \% \\
\text { Level } 120 \%: x_{\text {mean }}=100.95 \\
S=2.95 \\
\text { RSD }=2.92 \%\end{array}$ \\
\hline $\begin{array}{l}\text { Intra-day precision } \\
\qquad n=5\end{array}$ & $\begin{array}{c}\text { Level } 50 \%: x_{\text {mean }}=1383.68 \\
S=40.8261 \\
\text { RSD }=2.95 \% \\
\text { Level } 100 \%: x_{\text {mean }}=2771.62 \\
S=118.6253 \\
\text { RSD }=4.28 \% \\
\text { Level } 150 \%: x_{\text {mean }}=3579.68 \\
S=51.0468 \\
\text { RSD }=1.43 \%\end{array}$ \\
\hline $\begin{array}{c}\text { Inter-day precision } \\
\qquad n=5\end{array}$ & $\begin{array}{c}x_{\mathrm{sr}}=2241.32 \\
S=44.94 \\
\mathrm{RSD}=2.01 \%\end{array}$ \\
\hline
\end{tabular}

$P$ - peak area; $c$-concentration; $r$-correlation coefficient; $S$-standard deviation; RSD - relative standard deviation (\%); $S_{a}$-standard deviation of slope; $S_{b}-$ standard deviation of intercept. 


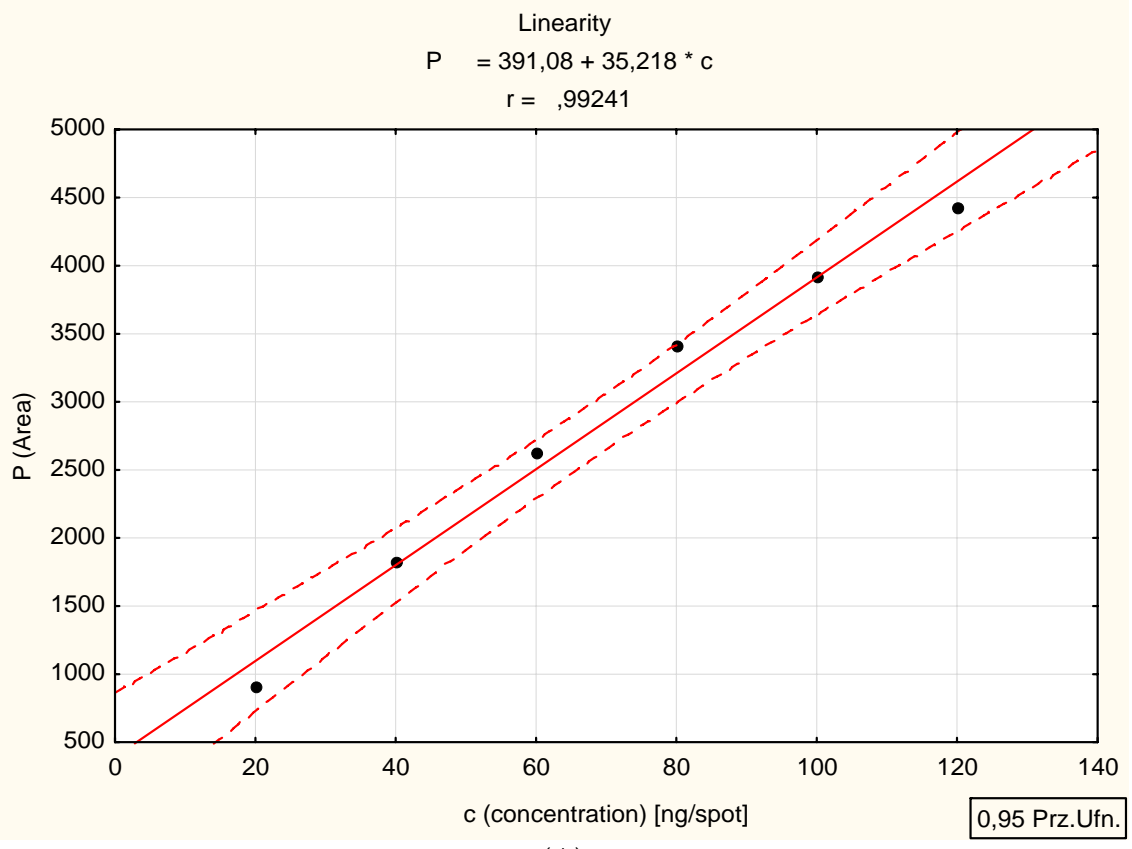

(A)

Histogram: residuals

Shapiro-Wilk W=,94204, $p=, 67568$

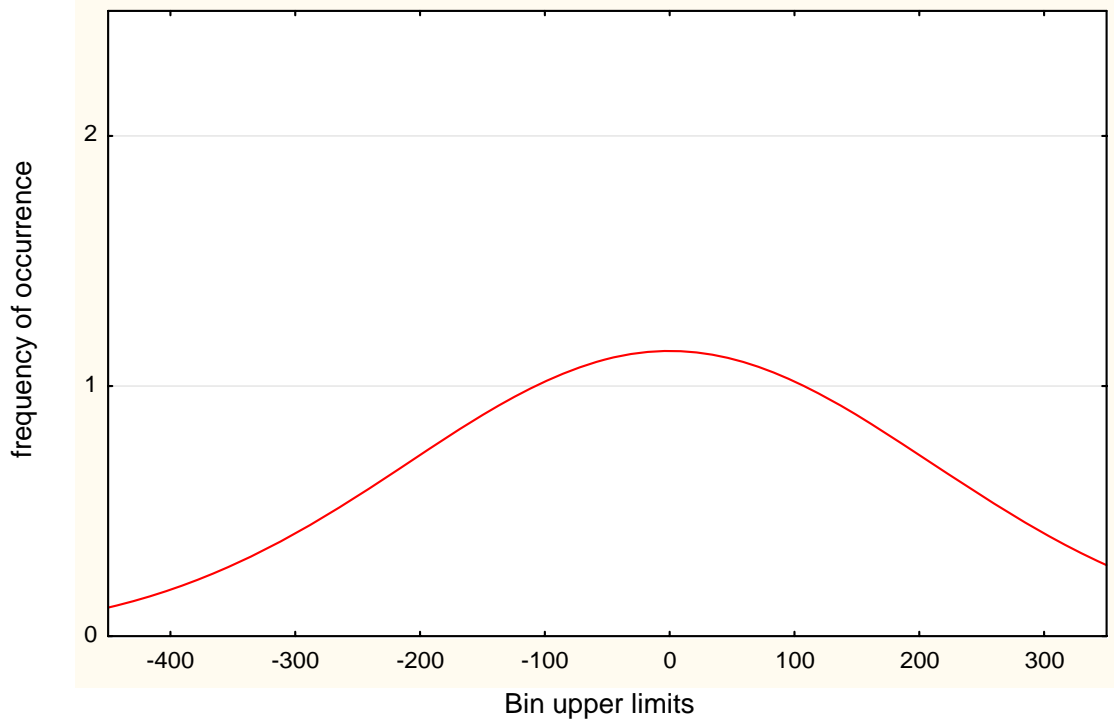

(B)

Fig. 2. Calibration plot (A) and plot of residuals (B) for sulfasalazine in the linear working range 
For quantitative analysis of sulfasalazine, peak areas for standard solution, and examined solutions were used. Validation of the developed method proved that it meets the acceptance criteria in the scope of specificity, sensitivity, linearity, precision, and accuracy (Table I).

The developed method is specific against sulfasalazine in a concentration range. For sample solutions used for quantitative analysis, no spots were found other than those of sulfasalazine. The measurement spots showed overlaying spectra, which indicates that there are no interferences.

The calibration curve for sulfasalazine was obtained by plotting the peak area versus concentration. It was found to be linear in the range 20$120 \mathrm{ng} \mathrm{spot}^{-1}$ (Fig. 2). The correlation coefficient $(r)$ was found to be $r=0.99$ showing good linearity. The adequacy of the regression model was examined by plotting the residuals. The obtained plots of residuals were without trends, which proves the linearity of the working range.

The developed method is sufficiently sensitive. The LOD and LOQ are both sufficiently low for the determination of low concentrations $17.11 \mathrm{ng} \mathrm{spot}^{-1}$ and $51.84 \mathrm{ng} \mathrm{spot}^{-1}$, respectively).

Precision studies were carried out at three different concentration levels, and the results of the intra-day and inter-day studies are presented in RSD within the range $1.43-4.28 \%$.

The recovery of the added standard to the sample was calculated, and it was found to be about $100 \%$, which indicated good accuracy of the method.

Under the developed method conditions, the small changes of analytical parameters do not have any significant influence on determined results.

Resolution and separation factors calculated for sulfasalazine and its impurities are satisfactory (Table II).

Table II. Resolution and separation factor for sulfasalazine and its impurities

\begin{tabular}{|c|c|c|}
\hline Examined peaks & Resolution $\left(R_{s}\right)$ & Separation factor $(\alpha)$ \\
\hline Sulfasalazine + imp 1 & 1.37 & 2.32 \\
\hline Sulfasalazine+ imp 2 & 1.19 & 1.90 \\
\hline Sulfasalazine $+\operatorname{imp~3~}$ & 1.02 & 2.37 \\
\hline
\end{tabular}

Absorption spectra registered for the impurities and sulfasalazine standard substance are similar with absorption maximum at $360 \mathrm{~nm}$. Extraction of impurities from the chromatographic silica gel and analysis of the extract by UPLC-MS/MS enabled to establish the chemical structures of the determined impurities that are presented in Table III. 
Table III. Impurities of sulfasalazine standard substance identified by UPLC-MS/MS

\begin{tabular}{|c|c|c|c|}
\hline$R_{\mathrm{F}}$ & {$\left[\mathrm{M}+\mathrm{H}^{+}\right]$} & Proposed structure & Name \\
\hline 0.56 & 250.12 & & $\begin{array}{l}\text { 4-Amino- } N \text {-(pyridin-2- } \\
\text { yl)benzenesulfonamide }\end{array}$ \\
\hline 0.61 & 335.21 & & $\begin{array}{l}\text { 2-Hydroxy-5-((4-(2-iminopyridin- } \\
1(2 H) \text {-yl)phenyl)diazenyl)benzoic ac- } \\
\text { id }\end{array}$ \\
\hline 0.75 & 399.16 & & $\begin{array}{l}\text { 2-Hydroxy-5-((4-(N-(pyridin-2- } \\
\text { yl)sulfamoyl)phenyl)diazenyl)benzoic } \\
\text { acid } \\
\text { (sulfasalazine) }\end{array}$ \\
\hline 0.88 & 355.15 & & $\begin{array}{l}\text { 4-((2-Hydroxyphenyl)diazenyl)-N- } \\
\text { (pyridin-2-yl)benzenesulfonamide }\end{array}$ \\
\hline
\end{tabular}

The proposed chromatographic procedure was applied for the quantitative determination of sulfasalazine in pharmaceutical preparations. The determined content of sulfasalazine in commercial pharmaceutical preparations matched with the label claim (RSD values $2.20 \%-3.39 \%$ ). The obtained results with the statistical estimation are presented in Table IV. 
Table IV. Determination of sulfasalazine and its impurities in pharmaceutical preparations

\begin{tabular}{|c|c|c|c|c|}
\hline \multirow{2}{*}{$\begin{array}{c}\text { Pharmaceutical } \\
\text { preparation }\end{array}$} & \multirow{2}{*}{$\begin{array}{c}\text { Determined } \\
\text { content }(n=5)\end{array}$} & \multicolumn{2}{|c|}{ Concentration of impurities $(n=5)$} \\
\cline { 3 - 5 } & & $\operatorname{Imp} 1$ & $\operatorname{Imp} 2$ & $\operatorname{Imp} 3$ \\
\hline Sulfasalazin & $x_{\text {mean }}=97.88 \%$ & $x_{\text {mean }}=2.62 \%$ & $x_{\text {mean }}=7.27 \%$ & $x_{\text {mean }}=1.38 \%$ \\
EN 500 mg & $S=3.3214$ & $S=0.0771$ & $S=0.1286$ & $S=0.0661$ \\
& $\mathrm{RSD}=3.39 \%$ & $\mathrm{RSD}=2.94 \%$ & $\mathrm{RSD}=1.77 \%$ & $\mathrm{RSD}=4.79 \%$ \\
\hline Salazopyrin & $x_{\text {mean }}=100.28 \%$ & $x_{\text {mean }}=2.67 \%$ & $x_{\text {mean }}=6.71 \%$ & $x_{\text {mean }}=1.74 \%$ \\
EN 500 mg & $S=2.2118$ & $S=0.0743$ & $S=0.1016$ & $S=0.0611$ \\
& $\mathrm{RSD}=2.20 \%$ & $\mathrm{RSD}=2.78 \%$ & $\mathrm{RSD}=1.51 \%$ & $\mathrm{RSD}=3.51 \%$ \\
\hline
\end{tabular}

In further analysis, the purity of the analyzed drugs by the presented method has been evaluated. Preparation solutions at a concentration of $1.0 \mathrm{mg} \mathrm{mL}^{-1}$ of sulfasalazine were analyzed by the TLC-densitometric method as described above. On the registered densitograms, additional peaks beside the peak of active substance of $R_{\mathrm{F}}$ values 0.56 for impurity 1 , 0.61 for impurity 2, and 0.88 for impurity 3 were observed (Fig. 3). The concentration of impurities was determined by the internal normalization method. Determined concentration of impurities is similar in both preparations. The obtained results are presented in Table IV.

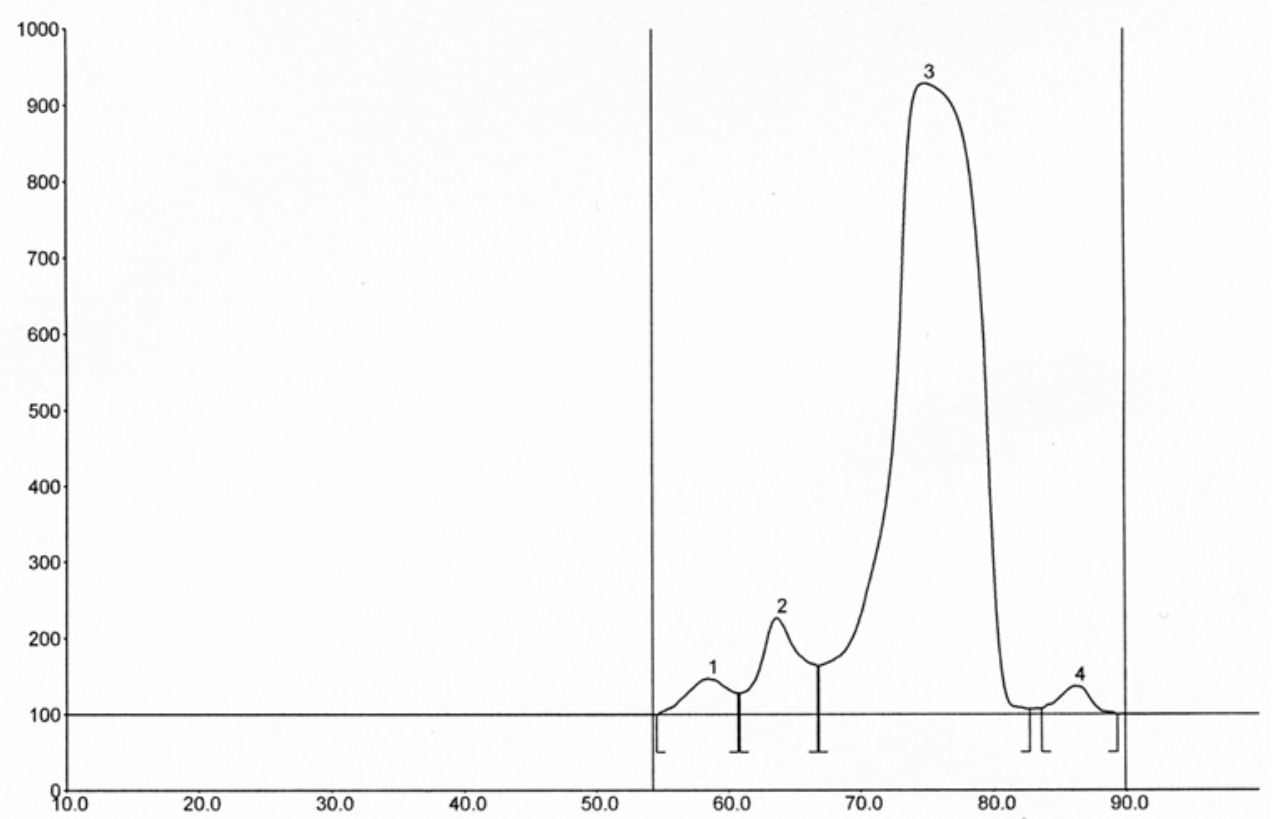

Fig. 3. Example densitogram for the purity determination of Sulfasalazin EN preparation registered at $360 \mathrm{~nm}$. Peaks 1, 2, and 4-impurities; peak 3-sulfasalazine 


\section{Conclusion}

This study indicates that the developed chromatographic-densitometric method presented in this paper is suitable for the determination of sulfasalazine in pharmaceutical preparations and for purity assessment of active substance beside possible impurities in pharmaceutical preparations. The developed method may be used as an alternative to pharmacopoeial methods.

\section{References}

[1] N. Pastor-Navarro, E. Gallego-Iglesias, A. Maquieira, and R. Puchades, Anal. Chim. Acta, 583, 377 (2007)

[2] Polish Pharmacopoeia, 9th edn., Warszawa, 2011

[3] G.L. Plosker and K.F. Croom, Drugs, 65, 1825 (2005)

[4] http: //www.liversupport.com/wordpress/2011/09/new-angle-for-tackling-liverfibrosis/

[5] R. Chauhan and S. Virmani, Int. J. Pharm. Sci. Rev. Res., 15, 120 (2012)

[6] N.M. El-Guindi, B.M. Abbas, R.I. El-Bagary, and E.A. Amer, J. Chem. Pharm. Res., 3, $412(2011)$

[7] G.Z. Gu, H.M. Xia, Z.Q. Pang, Z.Y. Liu, X.G. Jiang, and J. Chen, J. Chromatogr. B, 879, 449 (2011)

[8] S. Sadeghi, A. Motaharian, and A.Z. Moghaddam, Sensor. Actuat. B, 168, 336 (2012)

[9] G. Font, A. Juan-Garcia, and Y. Pico, J. Chromatogr. A, 1159, 233 (2007)

[10] ICH Guideline Q2(R1) on Validation of Analytical Procedures: Text and Methodology, International Conference on Harmonization, 6 Nov. 1996, incorp. Nov. 2005, Geneva

[11] K. Ferenczi-Fodor, B. Renger, and Z. Végh, J. Planar Chromatogr., 23, 173 (2010) 